\title{
PROBLEMAS DE IDENTIDAD DE LOS SISTEMAS JURÍDICOS
}

ara una teoría del derecho que parta de un enfoque sistémico, es de la máxima importancia resolver los problemas relativos a la identidad de los sistemas jurídicos. De ello depende tener respuestas satisfactorias a preguntas muy diversas, todas ellas relevantes desde el punto de vista jurídico. Por poner sólo algunos ejemplos: ¿cuándo dos normas jurídicas pertenecen al mismo sistema jurídico?; ¿qué criterios hacen posible distinguir un sistema jurídico de otro?; dado un golpe de Estado o una declaración de independencia, ¿están aún en vigor las leyes anteriores?, ¿puede el nuevo régimen o el nuevo Estado reclamar impuestos y deudas debidas al antiguo régimen o Estado?, ¿la persona que cometió un delito antes de haber ocurrido el cambio puede ser perseguida después de que este último haya ocurrido?. Éstas y otras cuestiones de parecida índole conforman la problemática de la identidad de los sistemas jurídicos.

Sin embargo, antes de resolver tales cuestiones es indispensable depurar el aparato conceptual que suele utilizarse. De este modo será posible plantear con mayor claridad y rigor los distintos problemas que se esconden tras el rótulo genérico de «la identidad de los sistemas jurídicos».

Para empezar, la expresión «sistema jurídico» es ambigua, ya que es empleada por distintos autores, y aun por un mismo autor, con significados diversos. Puede ser útil distinguir entre estos significados si no se quiere incurrir en confusiones.

Por ello, a continuación propondré diferenciar cuatro niveles en los que se pueden plantear problemas de identidad (sistema jurídico, orden jurídico, orden estatal, orden internacional) y dos perspectivas desde las cuales se pueden contemplar (sincrónica y diacrónica). Combinados los niveles con las perspectivas se obtienen ocho ámbitos distintos, cada uno de ellos con su particular problemática. 
Estas distinciones servirán de punto de partida para mostrar la plausibilidad de las tesis de este trabajo:

a) No hay un único problema de identidad de los sistemas jurídicos que requiera una única respuesta. Hay en realidad varios problemas, relativos a los distintos ámbitos, que merecen varias respuestas.

b) La problemática planteada a cada nivel y desde cada perspectiva no es siempre independiente de la de otros niveles y perspectivas, lo cual permite postular ciertas prioridades conceptuales entre las distintas respuestas.

\section{Niveles de sistema jurídico}

De lo notable que es la confusión originada en tomo al significado de «sistema jurídico» da fe la variedad de términos que se utilizan para describir una misma situación y la variedad de fenómenos que son descritos a veces con la misma expresión. Así, se habla de sistema jurídico, orden, ordenamiento, derecho, etc., refiriéndose a un conjunto de normas vigentes en un momento determinado, a la sucesión de esos conjuntos a lo largo del tiempo, etc. Resulta, pues, imprescindible hacer las oportunas matizaciones con el fin de especificar en qué sentido se utilizarán aquí determinadas expresiones.

\section{A) Sistema jurídico}

Una de las acepciones más usadas de «sistema jurídico» es la que lo concibe como un conjunto de normas. En este sentido, un sistema jurídico es el conjunto de normas jurídicas que tienen una determinada propiedad $P$ en un tiempo $t$ y en un espacio e determinados $^{1}$. La propiedad $P$ puede ser la validez (en su multiplicidad de significa dos), la vigencia, la aplicabilidad, etc.

Si el sistema jurídico es concebido como un conjunto de normas, la identidad del conjunto estará definida por la identidad de sus elementos (las normas). Por eso, cada vez que se crea una nueva norma o se deroga una norma perteneciente al sistema jurídico, se obtiene un nuevo conjunto, distinto del conjunto originario ${ }^{2}$.

${ }^{1}$ La especificación espacial rara vez aparece en las formulaciones estándar, lo cual puede tener alguna explicación, como se verá más adelante al establecer la distinción entre orden jurídico y orden estatal.

${ }^{2}$ Alchourrón, C.; Bulygin, E.: «Sobre el concepto de orden jurídico», en Análisis lógico y derecho, Madrid, C. E. C., 1991, págs. 393-407. 
Puesto que la identidad del conjunto se define por la identidad de sus elementos, es imprescindible recurrir a algún criterio de identificación de normas. Entre los criterios lingüísticos, cabe postular un criterio sintáctico, semántico o mixto ${ }^{3}$. No voy a desarrollar estas distinciones que podrían ser el marco de referencia de la problemática a tratar en este «nivel». Se trata simplemente de subrayar que con la expresión «sistema jurídico»aludiré a este significado específico, es decir, a un conjunto de normas que cambia cada vez que cambia uno de sus elementos. Los problemas que se plantean en este campo serán, así, problemas de «primer nivel».

\section{B) Orden jurídico}

Según lo dicho en el apartado anterior, los sistemas jurídicos cambian en el tiempo con la incorporación de nuevas normas al conjunto y la supresión de otras pertenecientes con anterioridad al mismo. Pero suele decirse que, a pesar de tales cambios, el «sistema» de alguna manera sigue siendo el mismo. Esto posibilita hablar de «sistema jurídico» en otro sentido.

Así es como lo ha entendido Raz, el cual distingue entre sistema jurídico momentáneo («sistema jurídico», en la terminología aquí adoptada) y sistema jurídico (llamado a veces por el propio Raz «no momentáneo» o «continuo») ${ }^{4}$. Esta idea de Raz se puede entender de dos formas. En un sentido, coincide con lo que aquí llamaré «orden jurídico». Pero, en otro sentido, parece incorporar, sin distinguirlo del anterior, el significado de lo que aquí denominaré «orden estatal».

Para Raz, un sistema jurídico momentáneo es el conjunto de normas pertenecientes a un sistema jurídico en un momento determinado. De este modo, el «sistema jurídico continuo» (orden jurídico) puede verse como una sucesión de «sistemas jurídicos momentáneos» (sistemas jurídicos). El problema de la identidad del orden jurídico estriba en hallar la propiedad que comparten los distintos sistemas jurídicos que pertenecen al mismo orden jurídico. Pero, llegados a este punto, surge el problema de la relación entre los conceptos de sistema jurídico y de orden jurídico.

${ }^{3}$ Cfr. Bulygin, E.: «Dogmática jurídica y sistematización del derecho», en Análisis lógico y derecho, cit., págs. 465-484. Para un desarrollo reciente de estas cuestiones, cfr. Moreso, J. J.; Navarro, P. E.: Orden jurídico y sistema jurídico. Una investigación sobre la identidad y la dinámica de los sistemas jurídicos, Madrid, C. E. C., 1993.

${ }^{4}$ Cfr. Raz, J.: The Concept of a legal System. An Introduction to the Theory of legal System. Oxford, Clarendon Press, 2. ${ }^{\text {a }}$ ed., 1980, págs. 34-35. 
No queda claro en el esquema propuesto por Raz si esta relación es de pertenencia o de inclusión ${ }^{5}$. Sin entrar en discusiones, voy a tomar la visión estándar de Alchourrón y Bulygin sobre este punto.

Estos autores entienden la relación antedicha como una relación de pertenencia. En este caso, el orden jurídico es una secuencia de sistemas jurídicos, con lo cual cobra importancia la determinación de la propiedad $P$ que permita establecer cuándo un sistema jurídico pertenece a un determinado orden jurídico. En estas circunstancias, no sería correcto decir que una norma pertenece a un orden jurídico (puesto que la relación de pertenencia no siempre es transitiva), sino más bien que una norma pertenece a un sistema jurídico, el cual, a su vez, pertenece a un orden jurídico.

Pero con lo anterior no quedan totalmente resueltos los problemas en este segundo nivel. Es preciso realizar una ulterior distinción entre orden jurídico y orden estatal.

\section{C) Orden estatal}

Con frecuencia, la distinción de niveles se detiene una vez hechas las anteriores precisiones. Habría, así, para cada momento determinado un conjunto de normas que conformaría un sistema jurídico. Además, distintos sistemas jurídicos en distintos momentos pertenecerían al mismo orden jurídico. Pero, en realidad, en este segundo «nivel», se mezclan comúnmente dos cuestiones, con cuya separación puede ganarse en claridad ${ }^{6}$.

Por ejemplo, a veces se dice que el «Derecho español» constituye un orden jurídico distinto del «Derecho alemán». Al mismo tiempo, se afirma que una revolución significa una ruptura del orden jurídico. Y, en ocasiones, se sostiene que, a pesar de los cambios revolucionarios acaecidos en un determinado país, ello no es óbice para considerar que estamos ante el mismo sistema $\mathrm{u}$ orden jurídico. ¿Cómo pueden compaginarse tales afirmaciones, si es que todas ellas tienen sentido?

Una forma de encarar la cuestión puede ser la de identificar sin más «orden jurídico» y «orden estatal». En este caso, se entiende que la sucesión de sistemas jurídicos tiene lugar en un mismo terri-

${ }^{5}$ Cfr. Bulygin, E.: «Tiempo y validez», en Análisis lógico y derecho, cit., pág. 197.

${ }^{6}$ Sobre este punto ha llamado la atención Caracciolo, pero no ha extraído todas sus consecuencias (cfr. Caracciolo, R.: Sistema jurídico. Problemas actuales, Madrid, C. E. C., 1988, págs. 19 y 20). Últimamente Bulygin también ha indicado que podría ser útil hacer esta ulterior distinción entre niveles (Bulygin, E.: «Algunas consideraciones sobre los sistemas jurídicos». DOXA, 9, 1991, págs. 265-266). 
torio y que, por tanto, tales cambios no afectan al Derecho del mismo, entendido como un orden. Como dije antes, a diferencia de la localización temporal, la localización espacial raramente se menciona de forma expresa ${ }^{7}$. Sin embargo, es importante hacerlo. Precisamente da la impresión de que el orden estatal ofrece el marco en el que se encuadra la sucesión de sistemas jurídicos, $\mathrm{y}$, al mismo tiempo, permite separar el orden jurídico vigente en un territorio en un determinado momento de otro orden jurídico vigente en el mismo momento pero en un territorio distinto. Además, la identificación entre «orden jurídico» y «orden estatal» ocultaría diferencias que tal vez hayan de considerarse relevantes. En primer lugar, impide ver en los cambios de régimen (por emplear una terminología política) o en los cambios ilegales de la constitución (por utilizar terminología jurídica) una alteración del orden jurídico. Si no se realizan más distinciones, habría que concluir, por ejemplo, que en la España del siglo XX habría existido un único orden jurídico, que se remontaría siglos atrás.

Por eso parece de interés proceder a una ulterior distinción entre orden jurídico y orden estatal ${ }^{8}$. Así, se podría afirmar que en la España del siglo XX se han dado sucesivos órdenes jurídicos, correspondientes a otros tantos regímenes políticos (monarquía, república, franquismo, monarquía parlamentaria), pero que tales órdenes pertenecen todos ellos al Derecho español.

Lo que ocurre es que, en ocasiones, el inicio de un nuevo orden jurídico coincide con el surgimiento de un orden estatal (por ejemplo, la independencia de un Estado). Pero ello es una circunstancia histórica que para nada impide proceder a su distinción conceptual.

${ }^{7}$ Sí que lo ha hecho, por ejemplo, Caracciolo (cfr. Caracciolo, R.: Sistema jurídico. Problemas actuales, cit., p. 31).

${ }^{8}$ En muchas ocasiones se incurre en confusiones por no realizar debidamente esta distinción. Entre los muchos ejemplos que podrían traerse a colación, véase Finnis, J. M.: «Revolutions and Continuity of Law», (1973), en Simpson, A. W. 8. (ed.): Oxford Essays in Jurisprudence (Second Series), Oxford, O. U. P., 1978. Finnis se da cuenta de la ambigüedad sistemática en que se usa el término «revolución», pero no cae en la cuenta de que él incurre en otro tipo de ambigüedad al usar el mismo, término para significar, por un lado, el cambio de orden jurídico, y, por otro, el cambio de orden estatal. Ello sucede cuando, bajo el concepto genérico de «revolución», Finnis procede al análisis del «golpe de Estado» (págs. 46-50), y, a renglón seguido, examina la llamada «sucesión pacífica de Estados» (págs. 52-53). Hay que recordar, sin embargo, que suele entenderse por golpe de Estado un intento de cambiar un régimen político dentro de un Estado determinado. De ahí que parezca recomendable tratar el problema de la independencia de un Estado (que afecta al orden estatal) como una cuestión distinta y previa conceptualmente a la de cambio de régimen político (que afecta al orden jurídico). 


\section{D) Orden internacional}

El análisis podría detenerse en este punto. Ello supondría tomar el orden estatal como un nivel independiente. Sin embargo, lo sostenido por ciertos autores permite plantear la posibilidad de un cuarto «nivel», que correspondería al orden internacional. El caso más representativo de la concepción del orden internacional como «sistema» es el de Kelsen". No obstante, a diferencia de lo que ocurre en los anteriores niveles, no parece tener sentido, en el lenguaje de los juristas, entender el orden internacional como sucesión de Estados. Por este motivo, como más tarde indicaré, los problemas relativos a la identidad del orden internacional pueden tratarse de forma independiente de los otros niveles.

\section{Perspectiva diacrónica o sincrónica}

Además de la distinción entre distintos niveles, es necesario distinguir entre dos diversas perspectivas. Así, los problemas de identidad pueden plantearse diacrónica o sincrónicamente. Desde la perspectiva diacrónica, se alude a la sucesión de sistemas dentro de un mismo orden jurídico, o a la sucesión de órdenes jurídicos dentro de un mismo orden estatal. Se toma como constante el elemento espacial y la variable que se tiene en cuenta es la temporal. En todo caso, se trata de establecer cuáles son las condiciones necesarias y/o suficientes para que pueda hablarse de cambio de sistema jurídico, de orden jurídico o, en su caso, de orden estatal.

Desde la perspectiva sincrónica, las preguntas que pueden formularse son cómo distinguir entre sí distintos sistemas jurídicos, órdenes jurídicos u órdenes estatales vigentes en el mismo intervalo de tiempo.

\section{Niveles y perspectivas: la dificultad del criterio único}

Si se combinan los niveles con las perspectivas mencionadas, surge en teoría la posibilidad de plantear dentro del tema genérico de la identidad de los sistemas jurídicos ocho posibles cuestiones. A continuación enumeraré del 1 al 4 los distintos niveles y añadiré la

${ }^{9}$ Cfr. Kelsen, H.: «Der Begriff der Rechlsordnung» (1958); tr. cast.: "El concepto de orden jurídico”, en Contribuciones a la teoría pura del Derecho. México, Fontamara, 1991, págs. 91-103. 
letra $\mathrm{D}$ o $\mathrm{S}$, según se contemplen respectivamente desde una perspectiva diacrónica o sincrónica. Así, se obtiene:

- 1D. En este caso se parte de un solo sistema jurídico y las cuestiones a formular son: en qué condiciones se mantiene el mismo sistema y cuándo cambia. Puesto que la identidad aquí depende de las normas que conforman el conjunto, mientras éstas no varíen, el sistema sigue siendo el mismo. Suele vincularse al criterio de legalidad, de tal forma que se entiende que el sistema se modifica con el ingreso o eliminación de alguna norma jurídica.

- 1S. Dentro de esta perspectiva, la hipótesis que suelen mantener los teóricos del derecho y los juristas en general es que hay varios sistemas jurídicos. La cuestión ahora estriba en ver por qué son distintos aquellos sistemas existentes en un mismo intervalo temporal. La primera respuesta obvia e inmediata es que son distintos porque también lo son sus respectivas normas jurídicas. Pero este criterio no resuelve totalmente la cuestión. Piénsese que, aunque empíricamente muy improbable, no está lógicamente excluido que existan sistemas con las mismas normas. Aún en estos casos, los juristas seguramente insistirían en que hay alguna diferencia entre ellos. En definitiva, el problema es que si no se toma en consideración el segundo o tercer nivel, habría que concluir que sistemas con las mismas normas jurídicas, aunque estén vigentes en territorios distintos, son idénticos. El único criterio que resta es diferenciarlos por su pertenencia a órdenes jurídicos distintos.

Alguien podría pensar que Kelsen ha resuelto este problema sin necesidad de acudir a otros niveles. Así, para este autor nunca se trataría de las mismas normas: el contenido de las normas siempre será distinto, porque aquél incorpora el ámbito de vigencia territorial. Sin embargo, esta contestación no resuelve el problema; simplemente, lo elude. La cuestión estriba precisamente en saber por qué ciertas normas tienen ámbitos de vigencia iguales y otras los tienen distintos. Y ésta es una pregunta que no puede ser contestada por las propias normas sin caer en circularidad. Sólo después de haber establecido criterios de identidad de unidades políticas independientes («orden estatal»), se pueden establecer distinciones entre normas que tienen distinto ámbito de vigencia.

- 2D. Lo que en este ámbito se plantea es saber qué variaciones en sistemas jurídicos sucesivos, manteniendo constante el espacio, son compatibles con la existencia del mismo orden 
jurídico; e, igualmente, qué variaciones en aquéllos dan lugar a un cambio en éste. Estas cuestiones suelen vincularse al criterio de la constitucionalidad.

Sin embargo, esto plantea algunos problemas. Dejando a un lado la dificultad de establecer cuándo un cambio es o no constitucional, puede mostrarse que la inconstitucionalidad de los cambios normativos no es ni condición suficiente ni necesaria del cambio de orden jurídico. No es condición suficiente, porque cabe pensar en cambios de normas que, aun producidos de forma inconstitucional, no suelen ser tomados como indicio determinante del cambio de orden jurídico (por ejemplo, la modificación inconstitucional de un reglamento municipal que regula materias de escasa importancia). Parece que, en estos casos, es preciso atender más a la importancia del contenido de la norma modificada que al carácter constitucional o no del procedimiento llevado a cabo. Tampoco se la puede considerar condición necesaria, porque casos en los que se está de acuerdo en que ha habido un cambio de orden jurídico, éste ha tenido lugar de forma «constitucional» (piénsese en el paso de la República de Weimar al régimen nazi en Alemania o el paso del régimen franquista a la Monarquía parlamentaria actual en España) ${ }^{10}$.

Cabe añadir que para contestar satisfactoriamente las preguntas relativas a $2 \mathrm{D}$, éstas deben formularse respecto a un intervalo de tiempo definido porque incluye al menos un cambio de sistema jurídico, lo cual implica un cambio, al menos, de norma jurídica.

- 2S. Una vez definido el intervalo temporal, entonces cabe preguntar: ¿Por qué se interpretan como distintos órdenes jurídicos simultáneamente vigentes en diferentes espacios? La vinculación que la contestación a esta pregunta tiene con el primer nivel ha sido puesto de relieve por muchos autores. Lo que no se ha percibido con tanta claridad es que la respuesta hay que buscarla en el tercer nivel: se consideran distintos porque pertenecen a órdenes estatales distintos. Ello, obviamente, no hace más que trasladar la pregunta de nivel. Así, pues, la identidad de los órdenes jurídicos parece depender,

${ }^{10}$ Para un tratamiento más detallado de estas críticas a los criterios «formales» de identidad del orden jurídico (entre los que cabría incluir, en mayor o menor medida, el criterio del soberano de Austin, el criterio de la constitucionalidad de Kelsen y el criterio de la regla de reconocimiento de Hart), cfr. Vilajosana, J. M.: El significado político del derecho. Identidad del orden jurídico y régimen politico. México, Fontamara, (en prensa). 
desde una perspectiva sincrónica, de la identidad de los órdenes estatales. De nuevo, decir que dos órdenes jurídicos son distintos en virtud del contenido de sus respectivos sistemas integrantes no es convincente. Existe la posibilidad lógica, aunque sea empíricamente muy improbable, de que existan órdenes jurídicos cuyos sistemas contengan las mismas normas. En estos casos, los juristas seguirían pensando que son dos órdenes distintos. Pero la diferencia ya no puede provenir de su distinto contenido, sino de la pertenencia a órdenes estatales distintos. Es una cuestión que no hay que desechar porque, de lo contrario, no se contesta a lo formulado en 1S. La suposición por parte de los juristas de que existen simultáneamente sistemas jurídicos distintos tiene como consecuencia la suposición de que, en un mismo intervalo temporal, coexisten órdenes jurídicos distintos. Por tanto, si bien el ámbito $1 \mathrm{~S}$ remite al $2 \mathrm{~S}$, en éste tampoco se resuelve la cuestión, ya que puede volver a formularse la misma pregunta que en $1 \mathrm{~S}$.

- 3D. A este nivel, se parte de un solo orden estatal y se interroga acerca de qué cambios de órdenes jurídicos son compatibles con la existencia continuada del mismo orden estatal y cuáles dan lugar a un cambio en éste. Esta cuestión se complica si se piensa en la posibilidad de que el cambio ilegal en el orden jurídico no sea una condición necesaria del cambio de orden estatal: puede otorgarse legalmente la independencia a un Estado. De nuevo, fijarse sólo en la constitucionalidad de los cambios no resuelve satisfactoriamente estas cuestiones. - 3S. El punto de partida ahora es la presencia de varios órdenes estatales. La pregunta a formular: ¿cómo se distingue un orden estatal de otro existente al mismo tiempo? ¿Hay que acudir de nuevo a un «nivel superior» para establecer la identidad en este sentido? Casos como el yugoslavo ponen de manifiesto que contestar a esta pregunta no es tan sencillo.

Por último, es menester realizar una precisión importante respecto al tercer nivel. Se trata de un nivel contingente. Con ello quiero decir que si bien en la actualidad nos sigue pareciendo importante aludir a distintas unidades políticas independientes, ello no tiene por qué ser siempre así. Si en el futuro se estableciera un único centro de poder internacional, cuyas decisiones vincularan efectivamente a todo el territorio que hoy se halla dividido en diferentes Estados, no tendría sentido hablar de este tercer nivel. Sí, en cambio, se podría seguir haciendo referencia a sucesiones de órdenes jurídicos dentro de un mismo orden internacional. 
- 4D. ¿Cuándo cambia el orden internacional? La respuesta que se dé a este interrogante depende de cómo se entienda el orden internacional. Cabe concebirlo como un conjunto de normas o bien como una sucesión de conjuntos. En el primer caso, ocurrirá algo parecido a lo que sucede en 1 D: el orden internacional variará a través del tiempo mediante la incorporación de nuevas normas o la eliminación de las ya existentes. En el segundo caso, se plantearán los mismos problemas que en $2 \mathrm{D}$, pero a nivel internacional. Lo que está claro es que, en cualquier caso, cuesta concebir al orden internacional como un cuarto nivel, ya que no parece posible entenderlo como sucesión de órdenes estatales. Más bien, la cuestión estriba en decidir si se toma como independiente el orden estatal o el orden internacional. A partir de ahí, cada uno de ellos constituiría un tercer nivel, con sus respectivos sistemas y órdenes jurídicos.

- 4S. Como se postula que el orden internacional es único, no parece tener sentido cuestionarse sobre su diferenciación respecto de «otros» órdenes internacionales.

Todo lo anterior pone de relieve la importancia de las cuestiones esbozadas y la dificultad en contestarlas. Al no realizar las distinciones precedentes, cabe entender que muchos autores utilizan un único criterio conceptual ${ }^{11}$ de identidad común a todos los niveles y perspectivas. Así, por ejemplo, el criterio del soberano de Austin, el de la norma básica de Kelsen o el de la regla de reconocimiento de Hart sirven explícita o implícitamente como criterios de identidad tanto de los sistemas jurídicos, como de los órdenes jurídicos o de los órdenes estatales y, en su caso (Kelsen), del orden internacional.

En cambio, al realizar las distinciones precedentes se percibe la necesidad de matizar mucho más en las respuestas. Utilizar el mismo criterio conceptual para contestar a los problemas de identidad en los distintos niveles y perspectivas oscurece distinciones relevantes.

\section{Prioridad conceptual}

Lo sostenido hasta ahora tiene ciertas implicaciones que pueden formularse en las siguientes tesis:

${ }^{11}$ Digo «criterio conceptual», porque de otro modo podría prestarse a confusión. Por poner un ejemplo, no afirmo (ni niego) que la misma regla de reconocimiento (tal como «es derecho lo que sanciona el rey en el Parlamento») sea utilizada para contestar todas las cuestiones que se plantean en el texto. Únicamente indico que el mismo concepto de regla de reconocimiento es utilizado para ese fin. 
1. A través de la combinación entre niveles y perspectivas, el tema genérico de la identidad de los sistemas jurídicos queda descompuesto en ocho posibles ámbitos de problemas. Estos ámbitos son:

- Sistema jurídico/perspectiva sincrónica (1S).

- Sistema jurídico/perspectiva diacrónica (1D).

- Orden jurídico/perspectiva sincrónica $(2 S)$.

- Orden jurídico/perspectiva diacrónica (2D).

- Orden estatal/perspectiva sincrónica $(3 S)$.

- Orden estatal/perspectiva diacrónica $(3 D)$.

- Orden internacional/perspectiva sincrónica $(4 S)$.

- Orden internacional/perspectiva diacrónica (4D).

2. Los teóricos del derecho y los juristas en general suelen estar interesados en comparar sistemas jurídicos pertenecientes a un mismo orden jurídico y órdenes jurídicos pertenecientes a un mismo orden estatal. Si ello es cierto, los distintos ámbitos no son totalmente independientes entre sí. Resolver satisfactoriamente algunas cuestiones relativas a un ámbito presupone haber resuelto antes la de otros.

3. Hallar (o presuponer) un criterio de identidad del orden estatal (3. ${ }^{\circ}$ nivel) es condición suficiente para resolver los problemas relativos a $2 S$. Así, dos órdenes jurídicos vigentes en el mismo intervalo temporal son distintos si pertenecen a dos distintas unidades políticas independientes.

4. Hallar (o presuponer) un criterio de identidad del orden jurídico (2. ${ }^{\circ}$ nivel) es condición suficiente para resolver los problemas relativos a $1 S$. Así, dos sistemas jurídicos vigentes en el mismo intervalo temporal son distintos si pertenecen a dos distintos órdenes jurídicos.

5. Hallar (o presuponer) un criterio de identidad del orden estatal (3. ${ }^{\circ}$ nivel) es condición necesaria (pero no suficiente) para resolver los problemas relativos a $2 D$. Se comparan órdenes jurídicos sucesivos que pertenecen al mismo orden estatal. Afirmar, por ejemplo, que la Constitución española de 1978 inauguró un nuevo orden jurídico distinto del vigente en el período franquista presupone tener un criterio que permita discernir cuándo dos órdenes jurídicos pertenecen a un mismo orden estatal. En ambos casos, se diría que son dos órdenes jurídicos determinados pertenecientes a un determinado orden estatal (en este caso el español) en distintos intervalos temporales.

6. Hallar (o presuponer) un criterio de identidad del orden jurídico (2. ${ }^{\circ}$ nivel) es condición necesaria (pero no suficiente) para resolver los problemas relativos a $1 D$. Se comparan sistemas jurídicos sucesivos que pertenecen al mismo orden jurídico. Por tanto, ni 
siquiera la pregunta acerca de cuándo dos normas pertenecen al mismo sistema jurídico puede contestarse sin tener (o presuponer) un criterio de identidad del orden jurídico (y, por lo dicho en 5, del orden estatal).

7. Los problemas relativos al ámbito $4 S$ no tienen sentido(a menos que hubiera algo así como un orden interplanetario).

8. Los problemas relativos a $4 D$ pueden ser tratados independientemente de los demás niveles, puesto que es difícil entender el orden internacional como sucesión de órdenes estatales.

9. El interés por hallar un criterio de identidad del orden estatal podría desaparecer si se creara efectivamente un orden internacional, sin unidades políticas independientes.

10. De 3, 4, 7 y 9 se infiere que si desapareciera el interés por la identidad al tercer nivel, carecerían de sentido los problemas relacionados con la perspectiva sincrónica a todos los niveles.

Si se aceptan las tesis anteriores, la resolución de los problemas de identidad del derecho entendido como «sistema jurídico» exige, desde el punto de vista conceptual, un determinado orden en su planteamiento. Dejando de lado el orden internacional, en primer lugar, es necesario contar con (o presuponer) un criterio de identidad del orden estatal. Una vez se ha logrado esto, se obtiene respuesta a las cuestiones que se plantean desde la perspectiva sincrónica a todos los niveles. Pero, además, se cuenta con un requisito necesario para plantear convenientemente los problemas relativos a la identidad del orden jurídico desde la perspectiva diacrónica, puesto que tener un criterio de identidad del orden estatal permite comparar ordenes sucesivos en el tiempo y pertenecientes al mismo orden estatal. En segundo lugar, tener un criterio de identidad del orden jurídico permite plantear convenientemente los problemas relativos a la identidad de los sistemas jurídicos, puesto que habilita la comparación de sistemas jurídicos sucesivos en el tiempo y pertenecientes al mismo orden jurídico.

Dicho de otro modo, en relación a las perspectivas tiene prioridad conceptual la perspectiva sincrónica, mientras que en relación a los niveles tienen prioridad conceptual los niveles superiores respecto a los inferiores.

La intención de este trabajo ha sido, pues, la de prevenir frente a dos ideas erróneas: pensar que un mismo criterio de identidad sea aplicable a todos los ámbitos en los que se puede dividir el tema genérico de la identidad del sistema jurídico, y suponer que los problemas de cada ámbito sean totalmente independientes de los demás.

\section{DOXA 17-18 (1995)}

